\title{
Effects of Timber Loads on Gross Vehicle Weight
}

\author{
Grzegorz Trzciński ${ }^{1} \bowtie$, Waldemar Sieniawski ${ }^{2}$,Tadeusz Moskalik ${ }^{1}$ \\ ${ }^{1}$ Warsaw University of Life Sciences, Faculty of Forestry, Department of Forest Utilization, Nowoursynowska 159, \\ 02-776 Warsaw, Poland, phone: +48 22 5938128, fax: +48 22 5938139, e-mail: grzegorz.trzcinski@wl.sggw.pl \\ ${ }^{2}$ University of Warmia and Mazury, Faculty of Environmental Management and Agriculture, Department of Forestry \\ and Forest Ecology, Pl. Łódzki 2, 10-727 Olsztyn, Poland
}

\section{Abstract}

Forest roads are characterized by low traffic, but heavy timber haulage trucks have a significant influence on road conditions and usually exceed the limit of $80-100 \mathrm{kN}$ per axle. The excessive weight of the trucks degrades the road surface and the forest environment. This article presents the results of a study of timber transport loads from their places of acquirement (forest districts) to the recipient (a large sawmill). The analysis includes the parameters of the timber transport vehicles and focuses especially on the gross vehicle weight (GVW), the total weight of empty truck and trailer during transport, and the weight of their loads. Scania, Iveco, Man, and Mercedes trucks with trailers and semitrailers designed for large-size pine timber transport were analyzed. ANOVA results revealed that all 80 analyzed vehicles exceeded the permitted GVW in the range from 2.28 to $19.94 \mathrm{Mg}$, transporting from 20.1 to $37.5 \mathrm{~m}^{3}$ of timber. The coefficient of determination between the amount of hauled timber and the GVW equaled $62.7 \%$. Research conducted previously concluded that forest roads with ground and gravel surfaces are not suitable for overloads of $120-150 \mathrm{kN}$ per axle. Therefore, it is necessary to find a logistical solution that will allow the load volume to be maintained at an acceptable level without destroying the forest road surface.

\section{KeY WORDS}

timber haulage, forest road, forest road loading, gross vehicle weight

\section{INTRODUCTION}

Timber transportation affects not only the forest environment and neighboring communities, but also the general public, because of all forest operations, only timber transportation has effects beyond the forest borders. Timber transportation is the most expensive part of the timber production process, and it becomes the factor that limits development of forests enterprises (Hamsley et al. 2007).
In 2008, Polish State Forests National Forest Holding (PGL LP) harvested more than 30 million cubic meters of timber; large-size timber constituted almost half of that amount (CSO 2009). The transportation of such large amounts of timber is a fundamental, but extremely complicated task. The timber is harvested in over 400 administrative units of PGL LP, and must be delivered to recipients spread throughout the country. The fact that transport operations are carried out by many small private companies makes this 
operation even more complicated (Paschalis-Jakubowicz 2008).

The necessity of transporting 30 million $\mathrm{m}^{3}$ of timber in Poland has impacts upon the condition of public and forest roads, and on traffic safety. Moreover, timber transportation consumes a large amount of fuel and emits greenhouse gases.

According to research from New Zealand, the use of a linear optimization algorithm can decrease the number of timber haulage trucks by $25-50 \%$ (Murphy 2003). The potential of achieving such a reduction while maintaining transport ability at the same level makes conducting scientific tests worthwhile (McDonald et al. 2001). Another study in Sweden showed that the optimization of routes can lead to a $22 \%$ reduction of empty runs (Arvidsson 1998). This has direct effects on another serious issue - fuel consumption. As Forsberg and Löfroth (2003) showed, fuel consumption is an important issue, and its costs may constitute around $25 \%$ of transport expenses. Additionally, the fuel consumption on forest roads may be up to $70 \%$ higher than on public roads. This situation is a specific interaction because timber transport has a direct impact on road surfaces, but the quality of the transport network is important in determining average speed and fuel consumption.

The location of the roads which provide access to the forest area (optimal density of the roads in $\mathrm{m}$ per ha), and the technical quality of those roads as determined by the project parameters (especially the surface construction and the road maintenance input), are the most essential factors, and are distinctive to the forest network (Olsson, Lohmander 2005; Žáček, Kleč 2008).

Most studies that determine the number of trucks carrying timber loads are not based on direct measurements, but rather on the basis of the capacity defined by the vehicle producer and traffic regulations (Anon 2005; Nevečerel et al. 2007). It is necessary to determine the actual timber loads delivered by haulage trucks. When we become acquainted with the specification of the vehicles, for example, the gross vehicle weight (GVW) and the timber loads, this will allow us to take them into account at the stage of the projecting and the construction of forest roads, which in turn guarantees a reduction in damage to the road surface (Hajek 1995; Burke 1995; Martin et al. 1999).
The main objective of this study was to determine the actual weight and volume of a single timber load hauled by high tonnage trucks and their GVW. These values were determined from information including the weight of the empty vehicle and the trailer (under operating conditions with technical liquids and additional equipment), and the weight of the hauled load. On the basis of preliminary analyses of delivery bills, it was assumed that the average timber load exceeds the limits, which implies that GVW limits are also exceeded. Because the timber haulage trucks transport various loads (i.e. different assortments and species of timber), this paper reports the results of an analysis of Scots pine timber loads of varying dimensions.

\section{MAterial AND MEthods}

To achieve the most representative conditions for timber transport, the research was conducted in one of the largest pine timber processing plant, where timber loads are received from all over the country. Each day the plant receives about $800-1000 \mathrm{~m}^{3}$ of various dimensions large-size timber diameter was between $0.14 \mathrm{~m}$ and $0.35 \mathrm{~m}$, max. length: $13.20 \mathrm{~m}$., or in the form of logs of either 4.40 or $8.80 \mathrm{~m}$ long. All haulage trucks were weighed with and without their loads during two days of field work.

\section{Characteristics of different brands and models of vehicles used for timber hauling}

The makes and models of the haulage trucks used to deliver timber as high tonnage vehicle sets (truck and trailer) were determined on the basis of both:

- fieldwork at the forest district management and at the timber recipient

- information taken from vehicle and trailer producers The initial review of timber haulage trucks provided characteristics of the following brands: Iveco, Renault, Volvo, Scania, Daf, Mercedes, and Steyer in addition to different types of trailers, including self-controlled trailers as well as those used to deliver timber. Only those trucks and trailers used for timber delivery were selected for study. The parameters of the haulage trucks that have influence on the selected aspects of roads quality, including the external dimensions (height, length, width, front and back overhangs), front and rear 
axle truck, and the truck weight together with the load weight and load characteristics were collected.

\section{Timber structure determination}

The delivery location was specified as the gate of the plant where timber is delivered, stored and processed. The suppliers were determined based on the delivery bills, and the addresses of the State Forest National Forest Holding administration units were assumed to be the locations of the suppliers. In that way we determined the nature of the timber delivered to a particular plant gate. For logistical reasons, timber is delivered by haulage truck sets consisting trucks and trailers. The distance travelled was calculated on the basis of the shortest distance between the forest district and the plant. Only roads with an axle pressure limit of above $8 \mathrm{Mg}$ were included.

\section{Measurement of the real weight of truck sets with and without the load}

At the plant, trucks and their respective loads were driven onto a calibrated, fixed platform scale and weighed. After a quality inspection and the unloading procedure, the empty set was reweighed. All trucks were driven onto the scale from the same direction. Every transporting set delivered timber 2-3 times a day and each time weighting was repeated in the same way. Every vehicle was weighed several so the real weight of the empty set was determined as a mean of all measurements. The difference in weight between the loaded and empty set gave the weight of the timber load.

\section{Statistical analysis}

ANOVA was used to determine if the weight or volume differences between trucks of one make and between trucks of different makes were statistically significant. The GVW, empty set weight, single load weight, and volume were analyzed to find a representative transportation set.

\begin{tabular}{|c|c|c|c|c|c|c|c|c|c|c|c|c|c|c|c|c|c|c|}
\hline 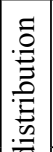 & $\mid$ & - & $z^{N}$ & $\stackrel{0}{\circ}$ & $\stackrel{\circ}{\circ}$ & 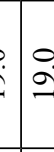 & $\dot{\varphi}$ & $\vec{a}$ & 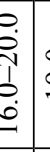 & $\stackrel{\circ}{\circ}$ & $\begin{array}{l}\circ \\
\stackrel{0}{-}\end{array}$ & $\stackrel{\circ}{2}$ & 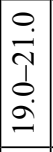 & 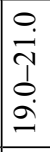 & $z^{+}$ & $\begin{array}{l}\dot{i} \\
\dot{\imath}\end{array}$ & $\stackrel{\circ}{\stackrel{i}{\sim}}$ & $\begin{array}{l}0 \\
\infty \\
\infty\end{array}$ \\
\hline 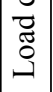 & 咅 & + & $\bar{z}$ & $\stackrel{\circ}{\infty}$ & ${ }_{\infty}^{0}$ & $\dot{b}$ & $\begin{array}{l}? \\
\end{array}$ & \begin{tabular}{c|c}
$r$ & 4
\end{tabular} & $?$ & $\vec{r}$ & $\stackrel{0}{\infty}$ & 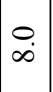 & $\begin{array}{l}0 \\
\infty\end{array}$ & $\stackrel{0}{\infty}$ & $z^{n}$ & 恣 & $\stackrel{n}{i}$ & \\
\hline $\mid \begin{array}{l}\frac{7}{50} \\
\frac{.00}{0} \\
3\end{array}$ & 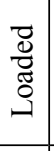 & + & & $\begin{array}{l}0 \\
\stackrel{\leftrightarrow}{v}\end{array}$ & $\stackrel{0}{\stackrel{\Xi}{े}}$ & 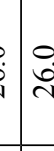 & $\overrightarrow{\dot{b}}$ & : & 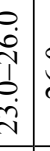 & $\begin{array}{l}0 \\
\dot{b}\end{array}$ & $\begin{array}{l}0 \\
\dot{D} \\
\ddot{i}\end{array}$ & $\left|\begin{array}{l}\dot{0} \\
\dot{b} \\
i\end{array}\right|$ & $\mid \begin{array}{l}0 \\
\dot{b} \\
i\end{array}$ & $\underset{\dot{D}}{\dot{i}}$ & & $\mid \begin{array}{l}n \\
\dot{q} \\
\dot{q}\end{array}$ & $\begin{array}{l}n \\
i \\
n\end{array}$ & $\stackrel{0}{\infty}$ \\
\hline 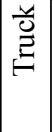 & $\begin{array}{l}\text { ते } \\
\text { : } \\
\text { | }\end{array}$ & - & & 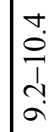 & $\begin{array}{l}3 \\
\stackrel{3}{1} \\
8\end{array}$ & $\vec{q}$ & ta & $y \mid \begin{array}{l}y \\
a \\
a \\
a\end{array}$ & 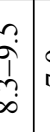 & $\stackrel{9}{r}$ & 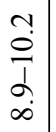 & \begin{tabular}{|c|} 
\\
$\dot{0}$ \\
0 \\
$\vdots$ \\
$\alpha$ \\
$\infty$ \\
$\infty$
\end{tabular} & 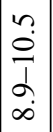 & 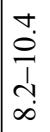 & & $\vec{\infty}$ & $\stackrel{\infty}{0}$ & $\underset{+}{\stackrel{P}{+}}$ \\
\hline $\begin{array}{l}0 \\
\tilde{Z} \\
\tilde{D}\end{array}$ & $\left|\begin{array}{l}\vec{\varpi} \\
\widetilde{\Xi} \\
\approx\end{array}\right|$ & 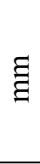 & $\widetilde{\jmath}$ & 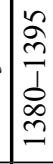 & 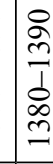 & 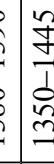 & 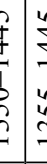 & 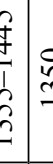 & $\begin{array}{c}\stackrel{2}{2} \\
n\end{array}$ & $\begin{array}{l}\stackrel{i}{n} \\
\stackrel{n}{n}\end{array}$ & 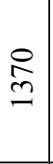 & 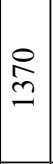 & $\begin{array}{l}n \\
m \\
2\end{array}$ & $\stackrel{n}{n}$ & & $\begin{array}{l}0 \\
\infty \\
-\infty\end{array}$ & $\begin{array}{l}0 \\
\infty \\
0\end{array}$ & 兽 \\
\hline$\frac{\vec{x}}{4}$ & $\mid \overrightarrow{0}$ & 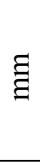 & ؟ & $\begin{array}{l}8 \\
\text { n } \\
n \\
1 \\
\text { D } \\
\text { ñ }\end{array}$ & $\begin{array}{l}8 \\
\infty \\
n \\
f \\
8 \\
n \\
n\end{array}$ & 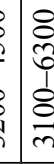 & \begin{tabular}{l|l}
$b$ \\
$b$ \\
$b$ \\
$b$ \\
$b$ \\
$b$
\end{tabular} & 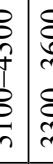 & 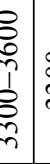 & 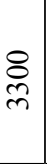 & $\begin{array}{l}8 \\
\stackrel{8}{n} \\
\hat{\delta} \\
8 \\
0 \\
0\end{array}$ & $\begin{array}{c}8 \\
0 \\
n \\
f \\
8 \\
i \\
n \\
n\end{array}$ & 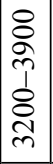 & $\begin{array}{l}8 \\
\delta \\
n \\
0 \\
\delta \\
n \\
n\end{array}$ & & 1 & 1 & 1 \\
\hline & 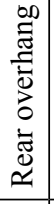 & 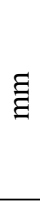 & $\stackrel{+}{ \pm}$ & $\mid \begin{array}{l}0 \\
\infty \\
\infty \\
\infty \\
\end{array}$ & 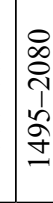 & 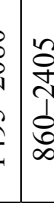 & 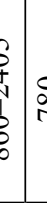 & $8 \mid$ & $\begin{array}{l}\frac{2}{2} \\
\frac{1}{2}\end{array}$ & $\stackrel{尺}{尺}$ & $\begin{array}{l}2 \\
\infty\end{array}$ & $\begin{array}{l}\approx \\
\infty\end{array}$ & $\stackrel{n}{\infty}$ & 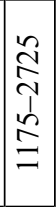 & & {$\left[\begin{array}{l}\infty \\
\infty \\
\sim\end{array}\right.$} & $\stackrel{\infty}{\infty}$ & \\
\hline 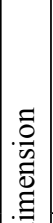 & 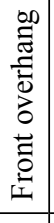 & 声 & క & $\stackrel{8}{ \pm}$ & 导 & $=\stackrel{2}{q}$ & $\begin{array}{l}f \\
f \\
f\end{array}$ & 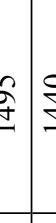 & 导 & 导 & 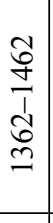 & 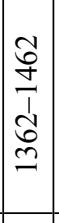 & $\begin{array}{l}\infty \\
\dot{\infty} \\
\pm\end{array}$ & 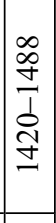 & & $\cong$ & $\cong$ & I \\
\hline 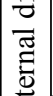 & $\frac{F}{5}$ & 吉 & in & $\begin{array}{l}\stackrel{\circ}{n} \\
i n\end{array}$ & $\begin{array}{l}\stackrel{n}{n} \\
\sim\end{array}$ & 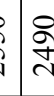 & 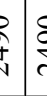 & 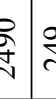 & $\underset{d}{\stackrel{g}{*}}$ & $\begin{array}{l}\stackrel{\infty}{+} \\
\stackrel{+}{\sim}\end{array}$ & 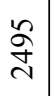 & 辛 & $\begin{array}{l}8 \\
0 \\
i\end{array}$ & $\begin{array}{l}8 \\
0 \\
i\end{array}$ & $\omega^{2}$ & $\begin{array}{l}0 \\
\tilde{n} \\
\end{array}$ & $\begin{array}{l}\circ \\
\\
\end{array}$ & $\begin{array}{l}\text { o } \\
\text { ñ } \\
\text { ñ }\end{array}$ \\
\hline 走 & 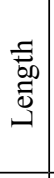 & 吉 & ــ & 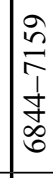 & $\begin{array}{l}\hat{\imath} \\
\tilde{\sigma} \\
\infty \\
\approx \\
\sim\end{array}$ & 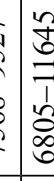 & 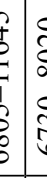 & 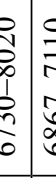 & $\begin{array}{l}0 \\
\frac{1}{1} \\
0 \\
0 \\
0\end{array}$ & $\begin{array}{l}8 \\
\& \\
\&\end{array}$ & $\begin{array}{l}\hat{n} \\
\frac{1}{1} \\
\hat{n} \\
\hat{b}\end{array}$ & $\mid \begin{array}{l}\hat{n} \\
2 \\
\hat{1} \\
\hat{0} \\
\frac{\pi}{2}\end{array}$ & $\begin{array}{l}\infty \\
0 \\
n \\
n \\
\infty \\
\infty \\
0 \\
0 \\
0\end{array}$ & 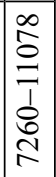 & $\Omega$ & $\stackrel{8}{\Xi}$ & $\begin{array}{l}8 \\
0 \\
0\end{array}$ & $\frac{8}{\ni}$ \\
\hline & 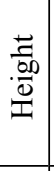 & 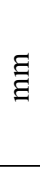 & $\Psi$ & $\begin{array}{l}n \\
\tilde{N} \\
\tilde{1} \\
\infty \\
0 \\
\tilde{n}\end{array}$ & $\begin{array}{l}\tilde{J} \\
\sim \\
\tilde{\jmath} \\
\delta \\
\bar{n}\end{array}$ & $\begin{array}{l}\frac{y}{n} \\
\frac{1}{n} \\
\frac{\pi}{n}\end{array}$ & 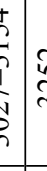 & $\begin{array}{c}c \\
\text { ch } \\
\end{array}$ & $\frac{2}{2}$ & $\stackrel{\vec{\sim}}{\sim}$ & 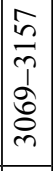 & 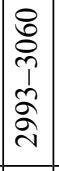 & $\begin{array}{l}\infty \\
\infty \\
N \\
n \\
\infty \\
\infty \\
\infty \\
\end{array}$ & 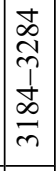 & $I^{2}$ & 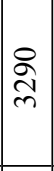 & $\begin{array}{l}\stackrel{2}{\text { }} \\
\text { d }\end{array}$ & \\
\hline 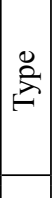 & 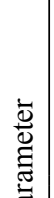 & 光 & 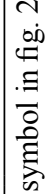 & 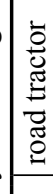 & 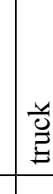 & $\frac{y}{0}$ & & 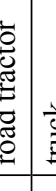 & 兯 & 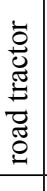 & 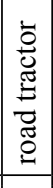 & 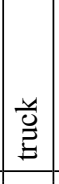 & 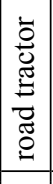 & 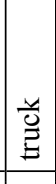 & & $\mid \begin{array}{c}\overrightarrow{1} \\
0 \\
8 \\
0 \\
\dot{\rho}\end{array}$ & $\begin{array}{l}0 \\
0 \\
0 \\
0 \\
0 \\
0\end{array}$ & 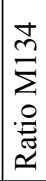 \\
\hline $\begin{array}{l}\vec{E} \\
\overrightarrow{\tilde{n}} \\
\bar{m}\end{array}$ & 2 & & 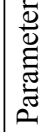 & $\begin{array}{l}8 \\
0 \\
\Xi\end{array}$ & $\begin{array}{l}8 \\
0 \\
己\end{array}$ & $\begin{array}{c}3 \\
\tilde{n} \\
\tilde{n}\end{array}$ & $5 \mid 0$ & 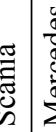 & 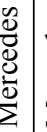 & $\begin{array}{l}\frac{0}{d} \\
\frac{d}{0} \\
\stackrel{0}{0} \\
\sum\end{array}$ & $\frac{8}{8}$ & $\frac{2}{2}$ & 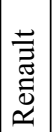 & 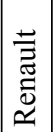 & 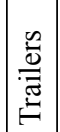 & & $\begin{array}{l}\text { Оे } \\
\text { ○ }\end{array}$ & \\
\hline
\end{tabular}


Statistical analysis of the results (GVW, the weight of set, the total timber volume and timber load) was performed with the computer program Statgraphics ${ }^{\circledR}$ Plus for Windows. Differences were recognized as significant when the test probability Po was lower than the assumed significance level $(\mathrm{Po}<0,05)$.

The following features were analyzed:

1. Characteristics of the results by groups (arithmetic mean, median, and standard deviation). A graphical interpretation is presented with box-and-whisker plots (fig. 3-6).

2. The differences between the averages within and between groups were determined with ANOVA. The hypothesis of whether significant statistical differences exist between the analyzed variables (groups) in the GVW and together with the load was tested. The different truck makes and different types of timber were taken into consideration. When such differences were identified the Fisher multiple range test was used.

3. The regression equation for the dependence of (GVW) and the load weight on the total timber volume for a single journey.

\section{Results}

\section{Characteristics of the trucks studied}

Timber was transported by four brands of trucks: Mercedes (types 3346, 2648, and 2653), Man (types 26361, 26462, 27402, and 33463), Iveco (type 260 E420), and Scania (types $124 \mathrm{C}$ and $143 \mathrm{H}$ ). The overall characteristics of the external dimensions are presented in the tab. 1 and fig. 1. There were various combinations of trucks and trailers - three-axle vehicles were paired to two-axle trailers, three-axle semitrailers with regulated lengths, two-axle semitrailers (fig. 1a), and two-axle trailers with regulated lengths (fig. 1b). The trucks observed had similar external geometrical dimensions, maximal gross vehicle weights and, when together with the load, had the same pressure on the axles.

\section{Analysis of timber transport structure}

Over the study period timber was delivered from 53 administration units of the State Forest, which were situated in 22 forest districts (fig. 2). A total of $4143 \mathrm{~m}^{3}$ of multidimensional pine timber was transported in

A

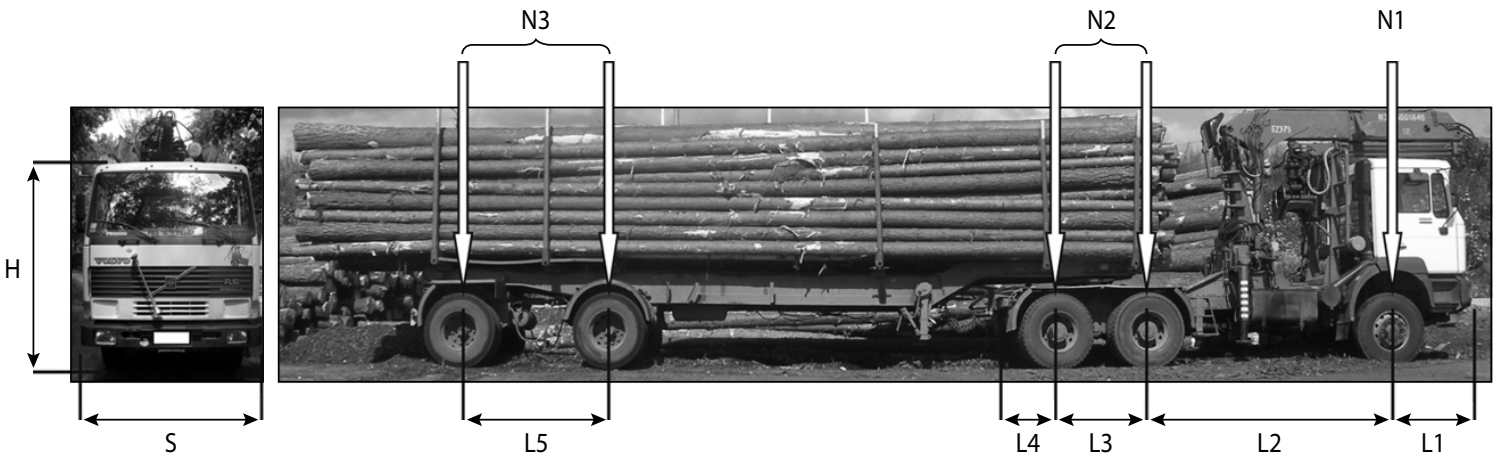

B
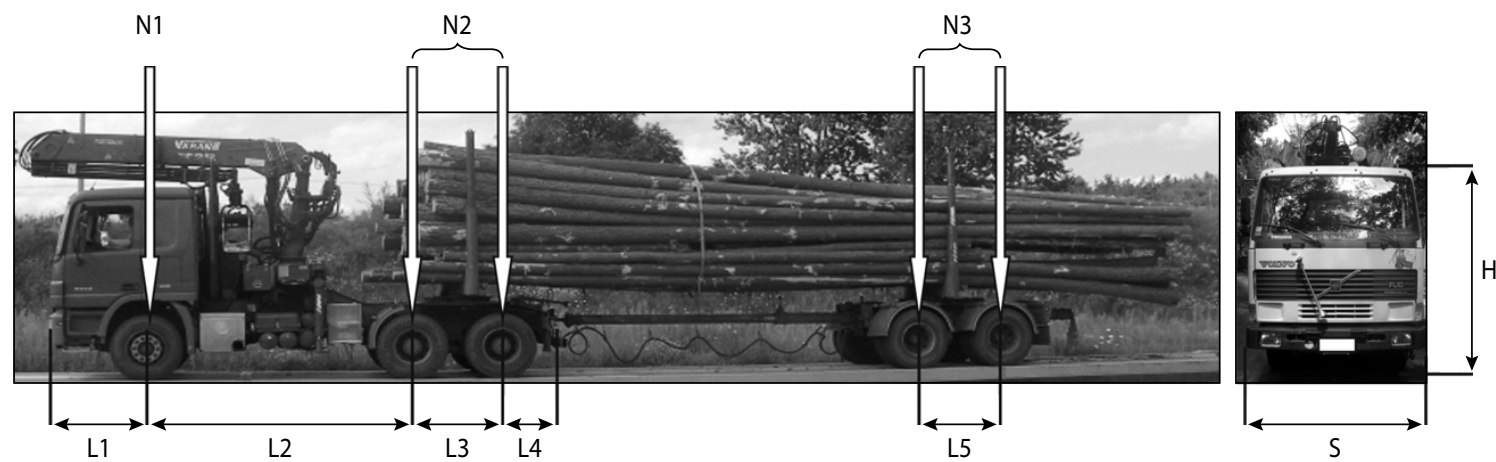

Fig. 1. Characteristic parameters of large-size timber haulage sets: A - with two-axle semitrailer, B - with two-axle trailer 

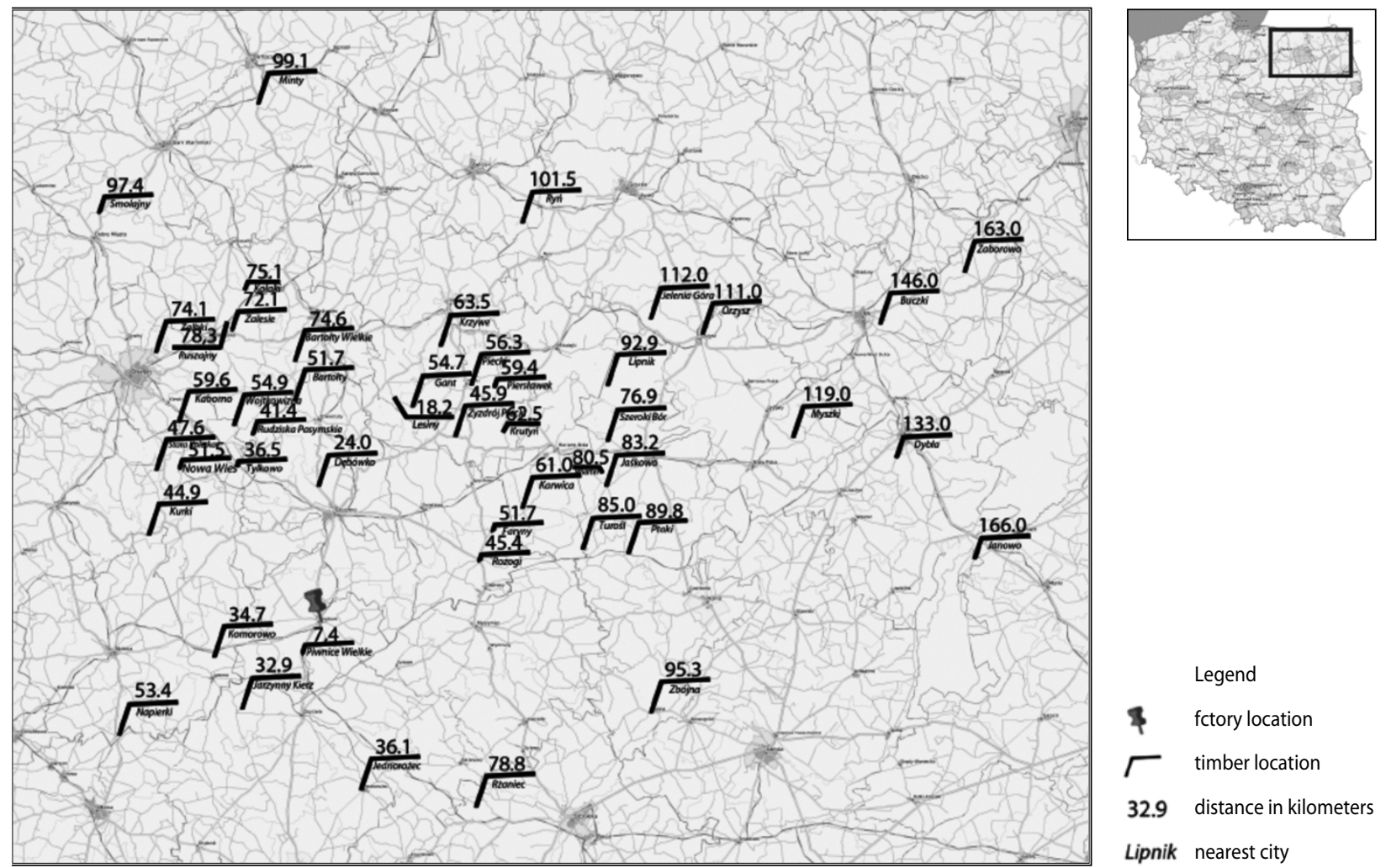

Fig. 2. Timber load distribution and receiver location

144 journeys. The shortest route recorded was between a timber yard located in the same forest district as the plant. That journey was nearly $7 \mathrm{~km}$ long. However, timber from that source accounted only for $2 \%$ of the total. The remainder was delivered from neighboring forest districts. These transport distances were between 7 and $165 \mathrm{~km}$. The average distance, weighted by the timber volume, was $72 \mathrm{~km}$.

The average load volume of the different makes of haulage trucks varied from 29.3 to $32.1 \mathrm{~m}^{3}$, and the range was between 20.1 and $37.4 \mathrm{~m}^{3}$ (fig. 3). It has been observed statistically significant differences between single loads and the average load of a particular truck set. Those loads were observed occasionally, and can be observed as outliers in fig. 3. The maximum timber load was above $34 \mathrm{~m}^{3}$ for Iveco and Man trucks, and $37 \mathrm{~m}^{3}$ for Mercedes and Scania trucks. The majority of the truck sets delivered timber loads from 28 to $32 \mathrm{~m}^{3}$. No statistically significant differences in the volumes delivered by different truck sets of different brands were observed, as determined via ANOVA.

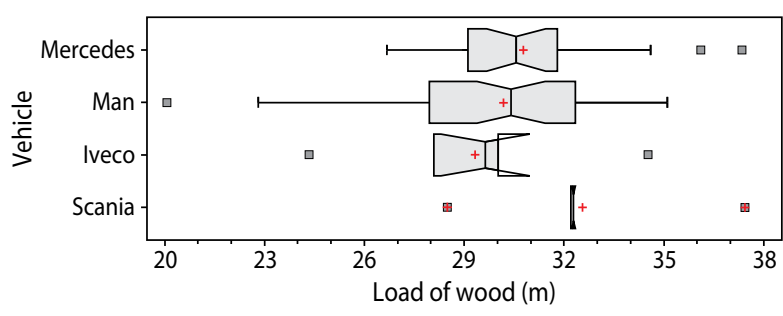

Fig. 3. Characteristics of single timber loads transported by haulage sets

\section{Real weights of the truck sets with and without loads}

A Man truck with $20.1 \mathrm{~m}^{3}$ of timber weighing $42.3 \mathrm{Mg}$ was the lightest set. Moreover, it was the smallest timber load of all. The heaviest load observed was also a Man truck set with a load of $34.0 \mathrm{~m}^{3}$ (fig. 4). The maximum and minimum $G V W$ of the Man truck sets were located outside the $95 \%$ confidence interval (fig. 4). The minimum $G V W$ of the Mercedes truck sets was $47.7 \mathrm{Mg}$ with $26.7 \mathrm{~m}^{3}$ of timber. The maximum $G V W$ for that brand 
(58.42 $\mathrm{Mg}$ ), was observed when the timber load was $33.1 \mathrm{~m}^{3}$. The Scania truck sets were the heaviest with an average weight of $55.26 \mathrm{Mg}$. The smallest range of GVW results was recorded for Iveco sets. They weighed between 46.62 and $51.80 \mathrm{Mg}$, with timber loads between 24.3 and $30.0 \mathrm{~m}^{3}$.

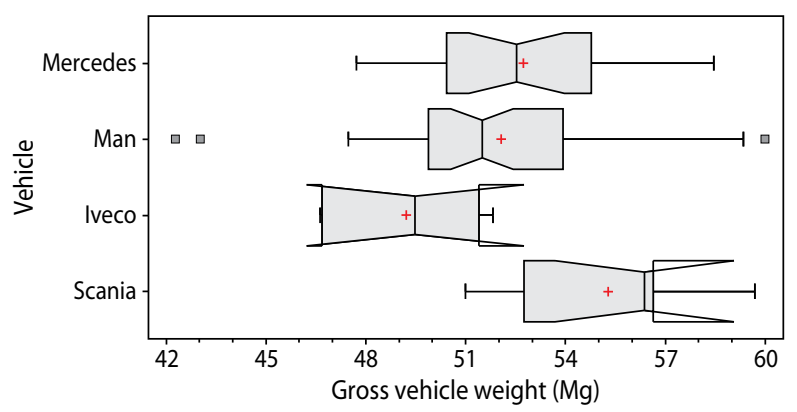

Fig. 4. Characteristics of the total weight of haulage sets $(G V W)$ with load

Significant differences in the weights of the truck sets between brands were identified by ANOVA. The following pairs of sets were found to have similar weights: Mercedes and Man, Mercedes and Scania, Man and Iveco.

The minimum weight of an empty truck set $(17.12 \mathrm{Mg})$ belonged to a Man truck, and the maximum weight, $23.12 \mathrm{Mg}$, was for a Scania truck set. Differences in the weights of empty truck sets were in the range of $2.00-5.00 \mathrm{Mg}$, with an average weight of between 19.70 to $20.80 \mathrm{Mg}$ (fig. 5). There were no statistically significant differences in the weights of empty truck sets of different truck makes and models.

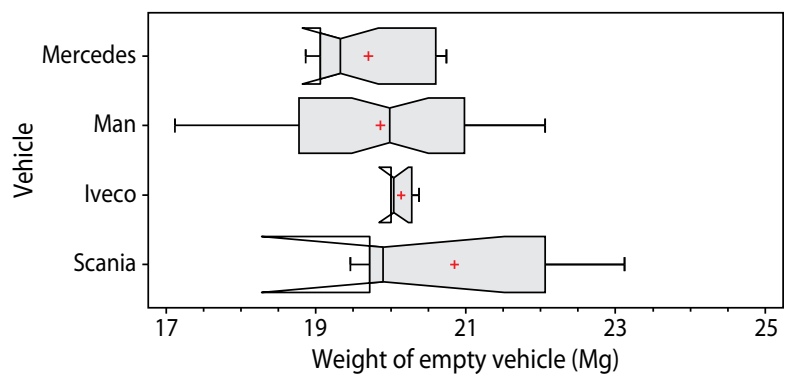

Fig. 5. Characteristic weight of empty haulage sets for largesize timber haulage

\section{Weight of a single timber load}

The weights of single loads were determined by recording the weight differences of truck sets before and after unloading. For loads of large-size pine timber, the minimum and maximum weight did not always correspond to the minimum and maximum volume. For Man trucks, the minimum load weight was $20.26 \mathrm{Mg}$ with $20.0 \mathrm{~m}^{3}$ of timber (minimum load volume). However, the maximum load weight was $38.38 \mathrm{Mg}$, when the volume of timber load amounted to $34.0 \mathrm{~m}^{3}$ (the maximum load equaled $35.1 \mathrm{~m}^{3}$ and weighed $37.90 \mathrm{Mg}$ ). The maximum load weight $(38.22 \mathrm{Mg}$ ) was observed for the Mercedes set, when the timber load volume was $30.7 \mathrm{~m}^{3}$ (fig. 6). The maximum timber load observed for both the Man and Mercedes sets weighed around $37.60 \mathrm{Mg}$. It is worth noticing that smaller (by $6.7 \mathrm{~m}^{3}$ ) timber load weighed more (by $0.78 \mathrm{Mg}$ ) in case of the Mercedes set. The average weight of the timber load for Man, Mercedes, and Scania sets was around $32.00-33.00 \mathrm{Mg}$, and $29.00 \mathrm{Mg}$ for Iveco (fig. 6). No statistically significant differences between the weights of loads delivered with different sets were observed.

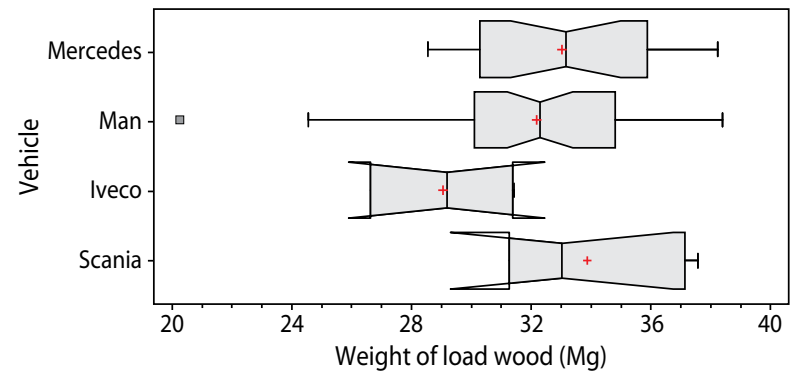

Fig. 6. Weights of single loads hauled by the analyzed sets

\section{Dependence of the total weight of the set and the load on the volume of the delivered timber}

There was no significant difference between the weight of the empty sets and volume of the loads. As a consequence, we decided to devise an equation for the whole weight of the truck set in relation to the volume of the transported timber and the weight of the empty truck. Based on the highest determination coefficient $\left(\mathrm{R}^{2}\right)$ and the lowest standard error of estimation, the following regression model has been designed: 


$$
G V W=\frac{0.001}{0.00000989876+0.00028079 T L^{-1}}
$$

where:

$G V W$ - gross vehicle weight, $\mathrm{Mg}$,

$T L \quad$ - timber load capacity, $\mathrm{m}^{3}$.

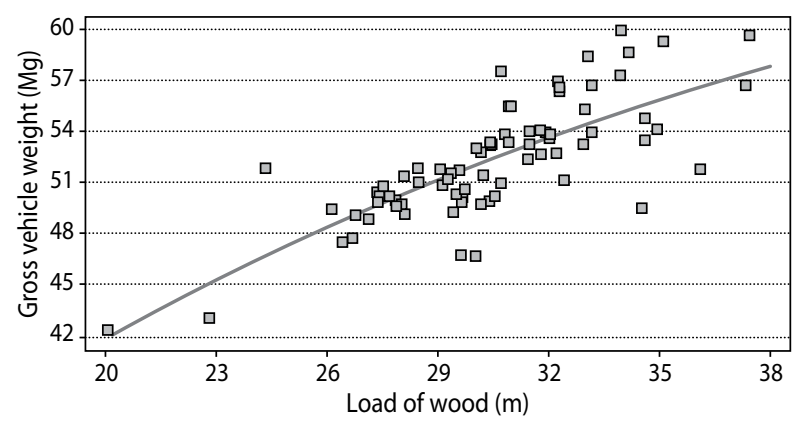

Fig. 7. Dependence of GVW on the load capacity

The volume of the transported timber determines the $G V W$. The determination coefficient $\left(\mathrm{R}^{2}\right)$ was estimated as $62.65 \%$.

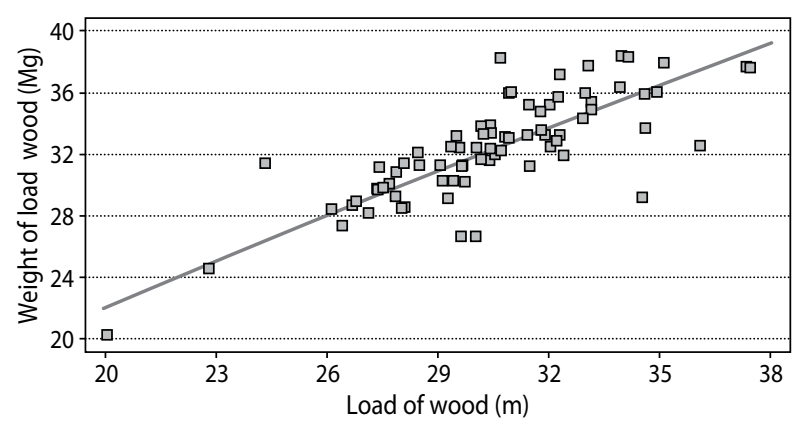

Fig. 8. Dependence of hauled load weight on the load capacity

The weight of a single load $(T W)$ was determined by the volume of the timber load $T L$, where $\mathrm{R}^{2}$ was equal to $69.19 \%$. The regression model for the weight of the timber load in relation to the load capacity was described with the following formula:

$$
T W=\frac{0.001}{0.00000346278+0.000837817 T L^{-1}}
$$

where:

$T W$ - timber weight, $\mathrm{Mg}$.
A graphical representation of both equations and input data are presented in figures 7 and 8 .

\section{Discussion}

In 1996 the EU member countries implemented directive 96/53/EC, which defines the acceptable weight limits of vehicles as 40.00 or $44.00 \mathrm{Mg}$ (depends on number of axle), and allows a single-axle pressure of $100 \mathrm{kN}$, and a two-axle pressure of $160 \mathrm{kN}$. Each country has an institution responsible for public transport and roads that has the authority to limit the gross vehicle weight. Moreover, it can also limit or increase the axle pressure in relation to certain EU regulations by specifying the roads that are suitable for such vehicles. An increased axle-pressure of up to $130 \mathrm{kN}$ is accepted in France, and an increased GVW is accepted in Sweden, Finland, and Norway (Department of the Environment 1997; FSVA 2001; Pravilnik... 2005).

All truck sets analyzed exceeded the acceptable total weight by $2.28 \mathrm{Mg}$ to $19.94 \mathrm{Mg}$, with an average overload of $12.02 \mathrm{Mg}$. The overloading of haulage trucks has been observed in countries other than Poland. In Ireland, McDonnell et al. (2008) claimed that $58-80 \%$ of vehicles exceed the acceptable total weight. Similar results were presented by Devlin (2008) who reported that $60 \%$ of vehicles exceeded the acceptable total weight. Moreover, $20 \%$ of them exceeded the maximum capacity defined by the producer. In research conducted in the USA (Hamsley et al. 2007), overloading of haulage trucks by $10 \%$ of the allowed weight was observed in 3-88\% of the vehicles.

The analyzed vehicles, grouped by makes had higher average GVWs by 5.60 to $5.80 \mathrm{Mg}$ (the empty vehicle) than the values obtained by Hamsley et al. (2007) (13.83 to $15.21 \mathrm{Mg}$ ). Such high empty vehicle weights (19.20 to $20.30 \mathrm{Mg}$ for the tested sets) automatically reduces the maximum possible load weight limited by EU regulations. With the average weight of a single timber load equaling $1.06 \mathrm{Mg}$, a haulage truck set is able to transport $22.6 \mathrm{~m}^{3}$ of timber (we take under the consideration the possible acceptable 5\% surplus, which can be the consequence measuring method employed by the control service). Besides, the study showed that $58 \%$ of trucks analyzed have transported more than $30 \mathrm{~m}^{3}$ of timber. If it's assumed that the maximum volume of a load cannot ex- 
ceed $22.6 \mathrm{~m}^{3}$, then considering that the average volume of a load observed in this study was $30.1 \mathrm{~m}^{3}$, and that the total volume of transported timber was $4.14 \mathrm{~m}^{3}$, and that it was transported over 144 journeys, then 40 additional journeys would be required. This would require 6657 additional kilometers of driving and the use of an additional 2148 liters of diesel, as well as an increase in carbon dioxide emissions of $1.50 \mathrm{Mg}$. On an annual scale, if plant production were at a level of $300000 \mathrm{~m}^{3}$, we would have to deal with 3347 additional journeys which would result in 482057 kilometers, diesel use on the level of 155573 liters (Arvidsson 1998), and carbon dioxide emissions of around $108900 \mathrm{~kg}$ (http://www2. rac.co.uk/route-planner/mileage-calculator/).

Martin at al. (1999) assumed that the great excess in $G V W$ s causes increased pressure on the vehicle axles and can result in the faster degradation and deformation of forest roads. This, in turn, will limit the accessibility of the area. It is necessary to take action to decrease the damage to forest roads. This can be achieved by increasing the number of the vehicle axles, decreasing the gross vehicle weight, using the regulation pressure in the tires CTI (Andersson, Granlund 1998; Granlund 2006), and arranging the load properly. Finally, we may temporally limit the vehicle entrance. Hamsley et al. (2007) gives advice and describes possibilities for controlling the timber recipients and limiting vehicle overloading, while at the same time using haulage truck sets more effectively and according to regulations regarding the acceptable load.

\section{Conclusions}

The location of the recipients and the suppliers together with information about the distance between them shows that the transport of timber mainly takes place on public roads.

The study indicates a significant overloading of timber haulage trucks. The average timber load volume equals $30.1 \mathrm{~m}^{3}$, which implies average overloading by $12.02 \mathrm{Mg}$.

Reducing the volume of transported timber to reduce the $G V W$ does not solve the timber transportation problem, because this will significantly increase the number of additional journeys, fuel consumption, $\mathrm{CO}_{2}$ emissions, and the environmental impact of transport.
A logistical solution to timber transportation problem, which allows the volume of single loads to be maintained at an acceptable level while satisfying EU regulations on $G V W$, and does not drastically increase the level of fuel consumption or number of additional journeys must be identified.

\section{Acknowledgements}

This work was supported by the Ministry of Science and Higher Education in 2008-2010 (grant number N N309298434).

\section{References}

Andersson G., Granlund P. 1998. Looking forward spring CTI. A study on timber-haulage vehicles equipped with CTI, spring 1997. SkogForsk Result, 2.

Antończyk S. 1989. Loads carried on forest roads and their variants. Sylwan, 133 (3), 53-60.

Arvidsson Per-Åke. 1998. Optimized route planning - good for both profits and the environment. SkogForsk Result, 23.

Burke D.F. 1995. Transportation logistics of timber both within forests and on non-national roads, Master of engineering science thesis, National University of Ireland, Dublin. http://www.ucd.ie/ foresteng/html/homepage/publications.htm (accessed February 2011).

Council Directive 96/53/EC laying down for certain road vehicles circulating within the Community the maximum dimensions in national and international traffic and maximum weights in international traffic (1996). Official Journal of the European Community, L235, 39.

CSO Regional and Environmental Surveys Division. 2009. Forestry 2009. Warsaw.

Department of the Environment 1997. Road traffic (construction, equipment and use of vehicles) regulations. Dublin. http://www.irishstatutebook.ie/1997/ en/si/0404.html (accessed 16.02.2011).

Devlin G. 2008. Applications and development of real-time GPS tracking systems and on-board load sensor technology for wood transport in Ireland Workshop: Developing cost-effective systems 
for wood procurement, harvesting and transport, COFORD 22 February 2008, Dublin Road, Portlaoise.

Fertàl D. 1994. Transport intensity in forest hauling roads. Správy Lesnického Výzkumu, 39, 34-37.

Forsberg M., Löfroth C. 2003. IT-study in four haulage rigs brings greater fuel economy with training and better roads. SkogForsk Result, 1.

FSVA. 2001. Richtlinien für die Standardisierung des Oberbaues von Verkersflachen. RSTO 01, Augsgabe.

Granlund P. 2006. CTI on roundwood haulage vehicles. Skogforsk Result, 3.

Hajek J. 1995. General axle load equivalency factors. Transportation Research Record, 1482, 67-68.

Hamsley A.K., Greene W.D., Siry J.P., Mendell B.C. 2007. Improving timber trucking performance by reducing variability of $\log$ truck weights. Southern Journal of Applied Forestry, 31, 12-16.

http://www2.rac.co.uk/route-planner/mileage-calculator/_(accessed on 21.03.2010).

Martin A.M., Owende P.M.O., O’Mahony M.J., Ward S.M. 1999. Estimation of the serviceability of Forest Access Roads. Journal of Forest Engineering, 10 (2), 55-61.

McDonald T.P., Taylor S.E., Rummer R.B., Valenzuela J. 2001. Information Needs for Increasing Log Transport Efficiency. Proceedings of First Inter- national Precision Forestry Symposium, Seattle, Washington, June 21-22, chapter 24.

McDonnell K.M., Devlin G.J., Lyons J., Russel F., Mortimer D. 2008. Assessment of GPS tracking devices and associated software suitable for real time monitoring of timber haulage trucks. In: COFORD Annual Report 2008, Director E. Hendrick, COFORD, Dublin, Ireland, 53-54.

Murphy G. 2003. Reducing trucks on the Road through optimal route scheduling and shared log transport services. Southern Journal of Applied Forestry, 27 (3), 198-205.

Nevečerel H., Pentek T., Pičman D., Stankić I. 2007. Traffic load of forest road as a criterion for their categorization - GIS analysis. Croatian Journal of Forest Engineering, 28, 27-38.

Olsson L., Lohmander P. 2005. Optimal forest transportation with respect to road investments. Forest Policy and Economics, 7 (3), 369-379.

Paschalis-Jakubowicz P. 2008. Limitations and barriers to the development of forest firms providing timber harvesting and transport services in Poland. Folia Forestalia Polonica, Series A - Forestry, 49/50.

Pravilnik o tehničkim uvjetima vozila u prometu na cestama, Narodne Novine 2005, 92, 1848.

Žáček J., Kleč P. 2008. Forest transport road according to natural forest regions in the Czech Republic. Journal of Forest Science, 54 (2), 73-83. 